\title{
A Unified Sustainable Manufacturing Capability Model for Representing Industrial Robot Systems in Cloud Manufacturing
}

\author{
Xingxing $\mathrm{Wu}^{1,2}$,Xuemei Jiang ${ }^{1,2}$,Wenjun $\mathrm{Xu}^{1,2}$,Qingsong $\mathrm{Ai}^{1,2}$,Quan $\mathrm{Liu}^{1,2}$ \\ ${ }^{1}$ School of Information Engineering, Wuhan University of Technology, China \\ ${ }^{2}$ Key Lab. of Fiber Sensing Technology and Information Processing, Ministry of \\ Education, China \\ wuxxwhut@163.com, jxm2001@whut. edu.cn, xuwenjun@whut. edu.cn, \\ qingsongai@whut.edu.cn, quanliu@whut.edu.cn
}

\begin{abstract}
Nowadays, the sustainable manufacturing capability of manufacturing devices has attracted more and more attention from academia and industry, in order to coordinate the conflicts between serious environmental impacts and economic benefits. As one kind of advanced manufacturing devices with intelligence, the industrial robot (IR) is an important driving force to make the production activities more efficient, safe and sustainable. A unified sustainable manufacturing capability model for representing IR systems in cloud manufacturing based on ontology was proposed in this paper, so as to solve the description problems in terms of the various capabilities of IR systems, and also to facilitate the factories to effectively manage the IR systems' manufacturing activities during the whole production life-cycle. The case study and its implementation show the developed ontology model is suitable for all types of IR systems, and can comprehensively reflects their sustainable manufacturing capabilities in real-time.
\end{abstract}

Keywords: Industrial Robot Systems; Sustainable Manufacturing Capability; Unified Model; Ontology

\section{Introduction}

Cloud Manufacturing is a new service-oriented manufacturing mode that provides user with on-demand manufacturing services after organizing manufacturing resources on the Cloud Manufacturing Service Platform on Internet. The virtualization of manufacturing resources, especially the manufacturing equipment, is the foundation of Cloud Manufacturing. IR is regarded as an advanced manufacturing equipment, which integrates mechanics, electronics, cybernetic, computer, sensor, artificial intelligence and other advanced technologies and it executes the tasks by manufacturing capability. Manufacturing capability is the level at which a manufacturing company or a manufacturing equipment completes a task during a specific manufacturing process [1]. It's not only the factor which influences an

adfa, p. 1, 2011.

(C) Springer-Verlag Berlin Heidelberg 2011 
enterprise to do related activities but also the preliminary judgment for users to select marketing partners. Current IR is becoming more and more complex and sophisticated. And an IR's manufacturing capability will vary with the occurrence of its failures and used hours. All kinds of reasons make it difficult for factories to effectively manage and maintain the manufacturing capabilities of IR systems. In addition, the sustainable manufacturing capability of an IR should be studied to solve current serious environmental problems due to the manufacturing model driven by economy. Sustainable manufacturing capability is a manufacturing equipment's ability to minimize the negative impacts on the environment and maximize the resource utilization ratio and comprehensive benefits during one or more life cycles of products [2]. But there is limited research in this area because IR is a new emerging technology industry.

In order to solve above problems, this paper is organized as follows. Section 2 introduced current researches about manufacturing capability. A unified sustainable manufacturing capability model for representing IR systems in cloud manufacturing was posed in section 3. In addition, the specific ontology structure was given too. In section 4, an IR individual was built to validate the theory proposed. Conclusions and future work were drawn in section 5 .

\section{$2 \quad$ Related Work}

At present, many scholars have done some researches on manufacturing capability. In 1969, Skinner firstly noted that manufacturing capability was determined by factors such as cost, quality and the relationships between the various elements [3]. From the point of view of an enterprise, Xun $\mathrm{Xu}$ et al. held that manufacturing capability was provided by software resources, human resources, material resources and processing resources (e.g., processing equipments, tools.) [4,5]. Only focused on manufacturing equipments, $\mathrm{Xu} \mathrm{W}$ et al. built their own capability models which included weight, type of machining parts, roughness, machining accuracy and feed efficiency [6,7].

Some scholars have studied the manufacturing abilities of IR systems. Nitzan D thought an IR achieved the functions by its actuators, sensors, computers and other auxiliary facilities [8]. Abele E studied the manufacturing precision which will be influenced by high load in the field of shaping, milling and cutting [9]. The manufacturing capability of an IR is determined by many factors such as cost, load capacity and processing interfaces $[10,11]$. Kahraman $\mathrm{C}$ et al. proposed a fuzzy multicriteria evaluation algorithm to analyze the manufacturing capability of an IR from the perspective of technical attributes and economic attributes [12]. Khandekar A et al. took seven indicators (e.g., installation location, repeatable positioning accuracy, payload, weight and speed of each joint.) into account to evaluate an IR's manufacturing capability [13]. Sen DK only think over some quantitative indicators [14]. In addition, there is an urgent need to reduce the energy consumption of an IR because of the shortage of resources and global warming $[15,16]$. Brossog $\mathrm{M}$ et al. proposed a modular model to analyze the energy consumption and dynamic behavior of an IR [17]. 
Current researches mainly focus on the manufacturing equipments and the manufacturing resources of enterprises. There are few studies about an IR's manufacturing capability, especially the sustainable manufacturing capability. And factories find it hard to grasp the manufacturing capabilities of IR systems with different functions and features. A unified sustainable manufacturing capability model for representing IR systems in cloud manufacturing based on ontology was proposed in this paper to solve above problems. And it's possible to dynamically improve the information of individuals in the ontology based on established mappings [6].

\section{A Unified Sustainable Manufacturing Capability Model for Representing IR Systems in Cloud Manufacturing}

IR is a manipulator with multiple joints or a mechanical device with multiple degrees of freedom. It automatically executes tasks by its own power and control mechanisms. It can accept human commands, and also can run according to pre-programmed procedures or the principles set by artificial intelligence techniques. In this paper, it was divided into point robots and continuous path robots according to control mechanisms. Point robots control an actuator from one point to another. They're used for spot welding, loading, unloading and handling. Continuous path robots make an actuator move at a given track. They're suitable for continuous welding and coating. On the basic of arms' movement modes, IR was classified into rectangular robots, cylindrical robots, spherical robots and joint robots.

\subsection{A Unified Description Model}

A unified description model of sustainable manufacturing capability for IR systems was proposed in Fig.1. There are five procedural steps as follows.

(a) The relation between an enterprise and an IR was set. As one kind of machining tool, an IR is possessed by an enterprise. An IR has its basic information which including name, ID, manufacturer and so on while an enterprise also has information which describes its name, phone and address.

(b) Various technical parameters (e.g., payload, memory capacity, repeatability.)of an IR is an important part of this model. They were collected from websites about IR systems. A suitable IR used for a specific task is selected by the values of its technical parameters. The manufacturing capability of an IR can be figured out through appropriate evaluation algorithms[18].

(c) In order to make this model dynamic, the manufacturing process of an IR was monitored. Operation is one part while the property during manufacturing is the other part. Operation can be extended according to the functions of an IR. An IR perform an operation on a task. And the property during manufacturing will reflect not only the execution status of a task but also the sustainability of an IR. This paper mainly took energy consumption and efficiency into account to analyze the sustainability of an IR. At the basis of power consumption, the 
consumption of resource used for producing power and the emission of carbon and dust particles exist in the process of generating power can be figured out.

(d) Mapping the sensory data to the property during manufacturing. Observation exists as a tie between sensors and properties. An observation is observed by a sensor. The value of a property is logged at a certain interval by an observation. The execution status of a task and the sustainability of an IR can be reflected in real-time through the mapping method. What's more, combining with the rules set in advance, it's helpful to expand and update the model .

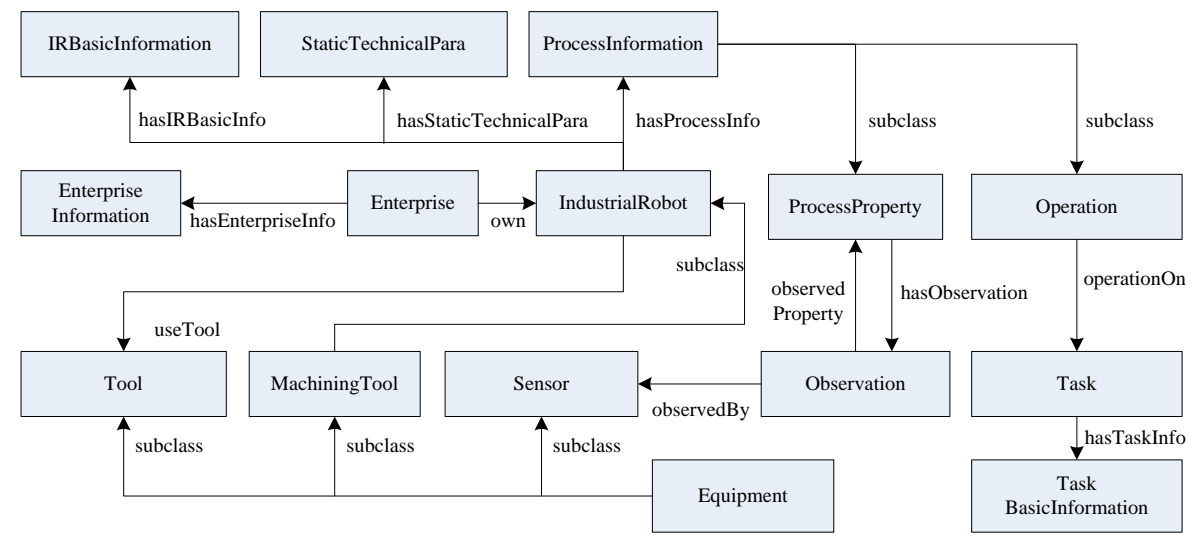

Fig. 1. A unified description model of sustainable manufacturing capability

The model proposed in Fig. 1 can be extended to meet the demands of a specific IR. In other words, it's fit for all kinds of IR systems. More relations will be detailed in next section.

\subsection{Ontology Structure}

An ontology model was proposed based on the unified description model. Ontology has many description languages. This paper adopted Web Ontology Language. And Protégé was used for ontology construction here.

The main concept in the ontology is "IR". It has three primary categories of information, namely:

- IRBasicInformation - describes the basic information of an IR, such as ID, name, type and manufacturer. The structure of an IR (e.g., movement mode of arm, type of power source.) was also included.

- StaticTechnicalPara-describes the manufacturing capability of an IR in terms of static attributes. ControlledAxes, MaxReach, MaxJointSpeed and Repeatability are classes which are expressed numerically while MountingPosition and ProtectionDegree are classes which are expressed qualitatively, as shown in Fig.2.

- ProcessInformation - reflects the status of an IR and a task in the process of manufacturing. It includes direct properties, the value of which is monitored by 
sensors, such as speed, temperature and power. There are also some indirect properties, whose value should be calculated or be reasoned, as shown in Fig.3. Properties, such as IRUtilizationRate, QualifiedRate, CycleTime, reflect the efficiency of an IR. And the power collected during the manufacturing illustrates the energy consumption of an IR. These properties are all factors of the sustainable manufacturing capability of an IR. Further, the state of an IR is split into five levels, namely, Off, Breakdown, Brake_On, Brake_Off, Running. This model will show the energy consumption under different states in real-time. Then the enterprise not only know the energy consumption of an IR, but also is able to figure out a management scheme of brake to minimize the energy consumption.

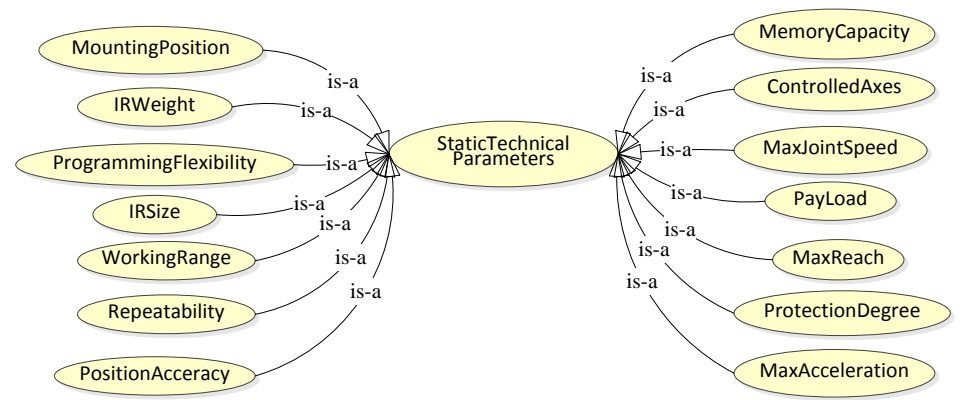

Fig. 2. Ontology of technical parameters

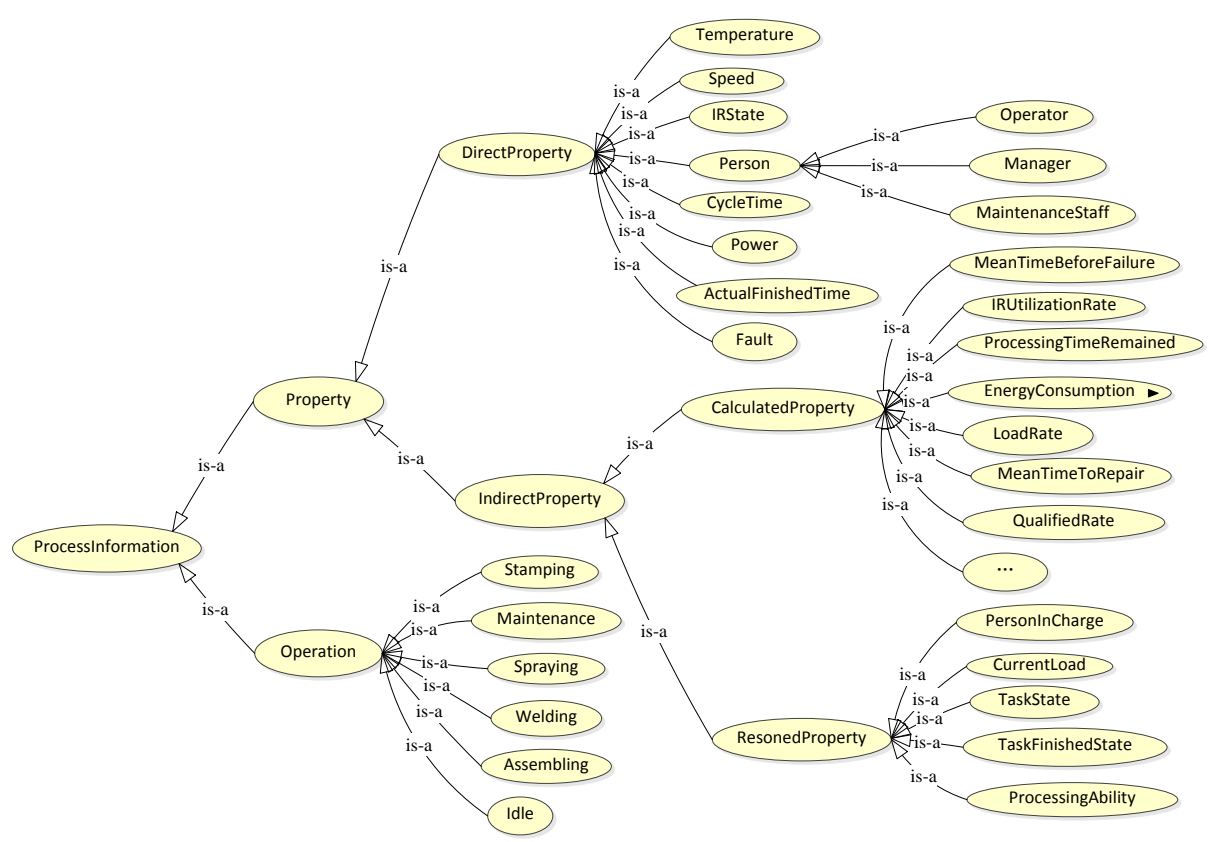

Fig. 3. Ontology of manufacturing process 


\section{$4 \quad$ Case Study and Implementation}

The semantic link between sensory data and model elements make it possible to analyze the sustainable manufacturing capability of an IR. An IR individual KUKAKR-16 arc HW was built to verify the theory mentioned above. It's a robot good at welding the door of a car. The query language SPARQL is used to seek the information of the model. Here is an example to query the energy consumption during the manufacturing, including at which time the observation happened, what's the value of the observation and what's the object of the observation, shown as follows. The values of other direct properties can be queried like that.

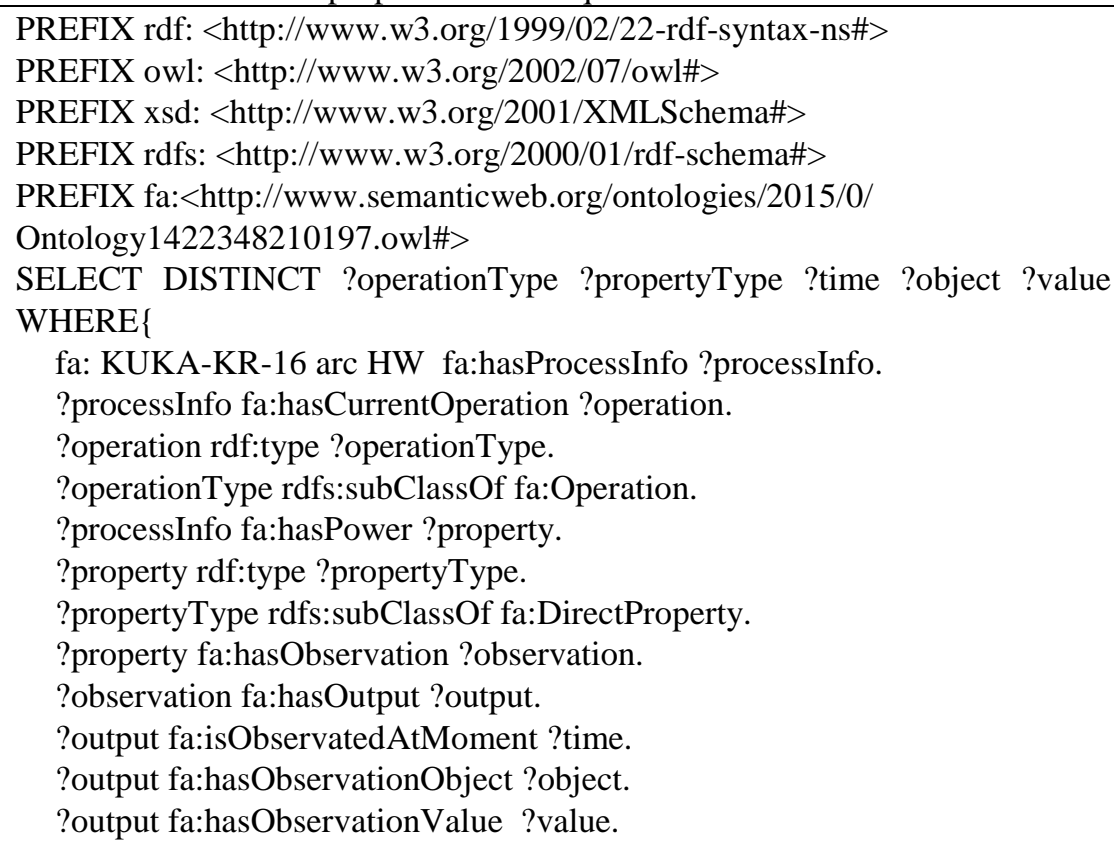

In addition, some inference rules were set as follows to help reasoning implied knowledge from real-time sensory data.

Rule1:Idle(?x),ProcessInformation(?y),hasIRState(?y,Brake_Off)-> hasCurrentOperation(?y, ?x)

Rule2:Idle(?x),ProcessInformation(?y),hasIRState(?y,Brake_On)->

hasCurrentOperation(?y, ?x)

These two rules means that if the current state of an IR is Brake_On or Brake_Off, the current operation of this IR is Idle.

At the basis of query results and inference outputs, it's possible to construct an analysis view composed of diagrams for each process property as depicted by Fig. 4. With these properties and relationships, the sustainable manufacturing capability of an IR is clearly shown in real-time. 


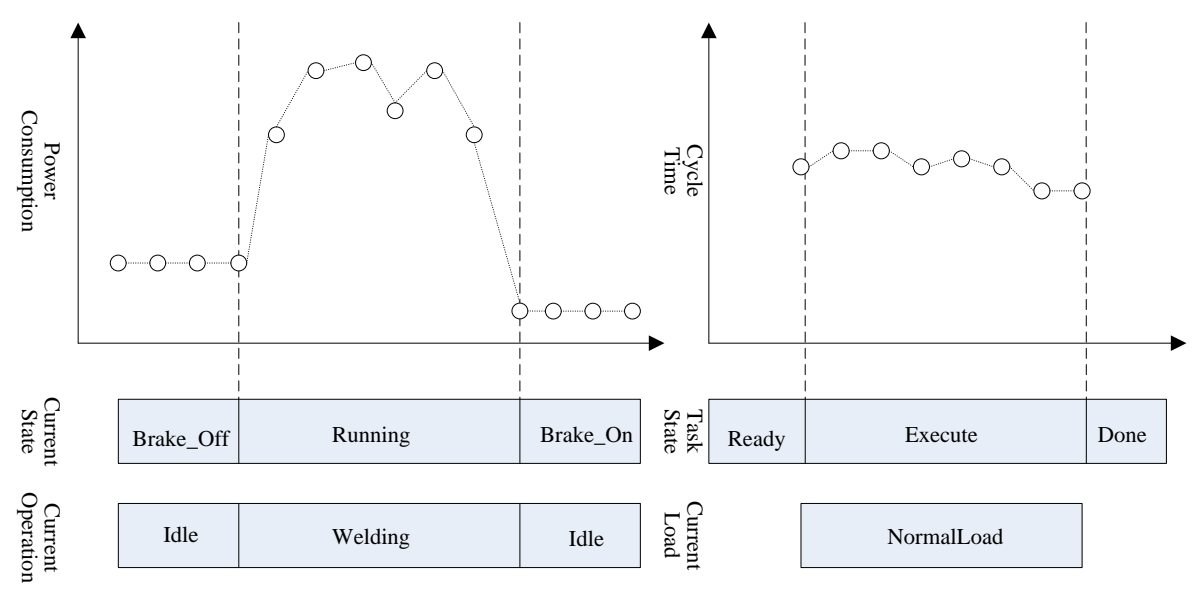

Fig. 4. Visualization of process property values

\section{$5 \quad$ Conclusions and Future Work}

A unified model of IR systems which can be described by ontology, is proposed to analyze the sustainable manufacturing capabilities of IR systems. It is able to solve the description problems due to the IR systems' various functions and complex structures. It is useful for factories to effectively manage the manufacturing capacities of IR systems. The proposed model still needs to be extended in future, and the evaluation algorithms should be studied to get a numerical result from the technical parameters and the manufacturing information.

\section{Acknowledgements}

This research is supported by National Natural Science Foundation of China (Grant No. 51305319), the Key Project of Natural Science Foundation of Hubei Province of China (Grant No. 2013CFA044), and the Wuhan International Scientific and Technological Cooperation Project (Grant No. 2014030709020306).

\section{References}

1. Luo Y, Zhang L, et al. A modeling and description method of multidimensional information for manufacturing capability in cloud manufacturing system[J]. The International Journal of Advanced Manufacturing Technology, 2013, 69(5-8): 961-975.

2. Smith R T, Melnyk S A. Green manufacturing[J]. Dearborn, MI: Society of Manufacturing Engineers, 1996: 3-4.

3. Skinner W. Manufacturing-missing link in corporate strategy[J]. 1969. 
4. Yuqian Lu,Xun Xu,Jenny Xu. Development of a Hybrid Manufacturing Cloud[J].Journal of Manufacturing Systems, 2014,33(4): 551-566.

5. Ameri F, Patil L. Digital manufacturing market: a semantic web-based framework for agile supply chain deployment[J]. Journal of Intelligent Manufacturing, 2012, 23(5): 1817-1832.

6. Xu W, Yu J, Zhou Z, et al. Dynamic Modeling of Manufacturing Equipment Capability using Condition Information in Cloud Manufacturing[J]. Journal of Manufacturing Science and Engineering, 2015.

7. Du L, Fang Y, He Y. Manufacturing Resource Optimization Deployment for Manufacturing Execution System[C]//ntelligent Information Technology Application, 2008. IITA'08. Second International Symposium on. IEEE, 2008, 1: 234-238.

8. Nitzan D. Development of intelligent robots: achievements and issues[J]. Robotics and Automation, IEEE Journal of, 1985, 1(1): 3-13.

9. Abele $\mathrm{E}$, et al. Modeling and identification of an industrial robot for machining applications[J]. CIRP Annals-Manufacturing Technology, 2007, 56(1): 387-390.

10. Rao R V, Patel B K, Parnichkun M. Industrial robot selection using a novel decision making method considering objective and subjective preferences[J]. Robotics and Autonomous Systems, 2011, 59(6): 367-375.

11. Chu T C, Lin Y C. A Fuzzy TOPSIS Method for Robot Selection[J]. The International Journal of Advanced Manufacturing Technology, 2003, 21(4): 284-290.

12. Kahraman C, Çevik S, Ates N Y, et al. Fuzzy multi-criteria evaluation of industrial robotic systems[J]. Computers \& Industrial Engineering, 2007, 52(4): 414-433.

13. Khandekar A, Chakraborty S. Selection of industrial robot using axiomatic design principles in fuzzy environment[J]. Decision Science Letters, 2015, 4(2): 181-192.

14. Sen D K, Datta S, Patel S K, et al. Multi-criteria decision making towards selection of industrial robot: exploration of PROMETHEE II method[J]. Benchmarking: An International Journal, 2015, 22(3).

15. Bornschlegl M, Drechsel M, Kreitlein S, et al. Holistic Approach to Reducing CO2 Emissions Along the Energy-Chain (E-Chain)[M]//Sustainable Automotive Technologies 2013. Springer International Publishing, 2014: 227-234.

16. Thiede S. Energy efficiency in manufacturing systems[M]. Springer Science \& Business Media, 2012.

17. Brossog M, Bornschlegl M, Franke J. Reducing the energy consumption of industrial robots in manufacturing systems[J]. The International Journal of Advanced Manufacturing Technology, 2015: 1-14.

18. Anant V.Khandekar, Shankar Chakraborty. Selection of industrial robot using axiomatic design principles in fuzzy environment[J]. Decision Science Letters, 2015,4(2):181-192. 